\title{
Long noncoding RNA are aberrantly expressed in human papillary thyroid carcinoma
}

\author{
MEILIU YANG ${ }^{1}$, JINLI TIAN $^{2}$, XIN GUO $^{2}$, YING YANG $^{1}$, RUHUA GUAN $^{3}$, MINGYUE QIU $^{1}$, YUKAI LI $^{1}$, \\ XUELING SUN ${ }^{2}$, YANFENG ZHEN $^{2}$, YAZHONG ZHANG $^{2}, \mathrm{CHUNYOU} \mathrm{CHEN}^{2}$, YANBING $^{2}$ and HUI FANG ${ }^{1,2}$ \\ ${ }^{1}$ Department of Internal Medicine, Hebei Medical University, Shijiazhuang, Hebei 050017; ${ }^{2}$ Department of Endocrinology, \\ Tangshan Workers' Hospital; ${ }^{3}$ Department of Medicine, Hebei United University, Tangshan, Hebei 063000, P.R. China
}

Received April 3, 2015; Accepted May 19, 2016

DOI: $10.3892 / 01.2016 .4653$

\begin{abstract}
Long noncoding RNAs (lncRNAs) have emerged as key regulatory molecules at almost every level of gene expression regulation. The altered expression of lncRNAs is a characteristic of numerous types of cancer, and lncRNAs have been demonstrated to promote the development, invasion and metastasis of tumors through various mechanisms. However, the role of IncRNAs in papillary thyroid carcinoma (PTC) remain unclear. In the present study, differentially expressed lncRNAs and mRNAs were detected by human lncRNA microarray in three pairs of PTC and adjacent noncancerous samples. The microarray results revealed that 675 lncRNAs and 751 mRNAs were abnormally expressed in the three PTC samples compared with adjacent noncancerous samples (fold change $\geq 2.0 ; \mathrm{P}<0.05$ ). To validate the microarray results, 8 differentially expressed 1 ncRNAs were randomly selected for quantitative polymerase chain reaction (qPCR). The results of qPCR were consistent with the microarray data; the 8 lncRNAs had an aberrant expression in the PTC samples compared with the adjacent noncancerous samples. Gene ontology and pathway analysis indicated that there were 7 downregulated pathways and 29 upregulated pathways in PTC. LncRNA classification and subgroup analysis revealed 7 pairs of enhancer-like lncRNA-mRNA, 9 pairs of antisense lncRNA-mRNA and 45 pairs of IncRNA-mRNA were differentially expressed between PTC and their paired noncancerous samples. In conclusion, the present study identified a series of novel PTC-associated lncRNAs. Further study with these lncRNAs is instrumental for the identification of novel target molecules that could lead to improved diagnosis and treatment for PTC.
\end{abstract}

Correspondence to: Dr Hui Fang, Department of Internal Medicine, Hebei Medical University, 361 East Zhongshan Road, Shijiazhuang, Hebei 050017, P.R. China

E-mail: fanghui@medmail.com.cn

Key words: papillary thyroid carcinoma, long noncoding RNA, microarray, cancer, mRNA

\section{Introduction}

In the majority of regions of the world, thyroid cancer incidence has increased over the last few decades (1). Papillary thyroid carcinoma (PTC) is the most common thyroid cancer, accounting for $85-95 \%$ of thyroid cancer (1-4). Potential risk factors for PTC include a family history of thyroid cancer, radiation exposure and abnormal iodine intake (4-7). In recent years, studies have demonstrated that genomic instability, alterations in epigenetic events and subsequent inappropriate gene expression are key in the regulation of PTC $(8,9)$. However, the mechanisms underlying PTC pathogenesis have not been elucidated.

Recent studies have revealed a complex involvement of epigenetic mechanisms in the regulation of gene expression, including the diverse activities of noncoding RNAs (ncRNAs) $(10,11)$. The roles of microRNAs have been relatively well studied in PTC, but other studies have revealed that alterations in PTC-associated long noncoding RNAs (lncRNAs) may lead to an altered regulation of key genes $(12,13)$. IncRNAs are transcript RNA molecules, $>200$ nucleotides in length, that do not encode proteins. IncRNAs are among the least well-understood of ncRNA species; however, they cannot be dismissed as transcriptional 'noise'. Thousands of lncRNAs have recently been described in mammals (14). Studies have revealed that an aberrant expression of IncRNAs results in abnormalities in gene expression and tumorigenesis. In addition, an altered expression of IncRNAs is a characteristic of numerous types of cancers, which have been revealed to promote the development, invasion and metastasis of tumors through various mechanisms (15-17). However, study concerning lncRNA involvement in PTC is in its infancy, and the identification of additional PTC-associated lncRNAs and their mechanisms of action require additional investigation.

The present study profiled the expression of lncRNAs and mRNAs in three paired PTC and adjacent noncancerous samples via microarray analysis, and identified a series of novel PTC-associated lncRNAs. In addition, the present study demonstrated that genes associated to these lncRNAs were associated with risk factors for tumorigenesis. The present results suggest that IncRNA expression patterns may provide novel molecular biomarkers for the diagnosis of and methods for treating PTC. 


\section{Materials and methods}

Patient samples. PTC samples and corresponding noncancerous tissue samples were prospectively obtained from 36 patients at Tangshan Workers' Hospital (Tangshan, China) between April 2014 and November 2014. The samples were snap-frozen in liquid nitrogen immediately following resection. Of these samples, three pairs were used for lncRNA microarray analysis and the remaining samples were used for additional validation in the present study. Patient characteristics are presented in Table I. The present study was approved by the Institutional Ethics Review Board of Tangshan Workers' Hospital, and all patients provided written informed consent.

RNA extraction. Total RNA was extracted using TRIzol ${ }^{\circledR}$ Reagent (Invitrogen ${ }^{\mathrm{TM}}$; Thermo Fisher Scientific, Inc. Waltham, MA, USA), according to the manufacturer's protocol. RNA quantification and quality were assured by NanoDrop ND-1000 spectrophotometer (Thermo Fisher Scientific, Inc. Wilmington, DE, USA). RNA integrity and gDNA contamination test were assessed by denatured agarose gel electrophoresis.

Microarray and computational analysis. Sample labeling and array hybridization were performed using the Agilent One-Color Microarray-Based Gene Expression Analysis (Agilent Technologies, Inc., Santa Clara, CA, USA), according to the manufacturer's protocol, with minor modifications. Briefly, mRNA was purified from total RNA following the removal of rRNA using a mRNA-ONLY ${ }^{\mathrm{TM}}$ Eukaryotic mRNA Isolation kit (Epicentre, Madison, WI, USA). Subsequently, each sample was amplified and transcribed into fluorescent cRNA along the entire length of the transcripts without 3' bias utilizing a random priming method (Flash RNA Labeling kit; Arraystar, Rockville, MD, USA). The labeled cRNAs were purified by RNeasy Mini kit (Qiagen, Inc., Valencia, CA, USA). The concentration and specific activity of the labeled cRNAs (pmol Cy3/ $\mu$ g cRNA) was measured by NanoDrop ND-1000. The labeled cRNAs were hybridized onto a Human LncRNA Microarray V3.0 (Arraystar), which is designed for 30,586 lncR NAs and 26,109 coding transcripts. The lncRNAs were carefully constructed using the following public transcriptome databases: RefSeq (NCBI Reference Seqeunce Database; www.ncbi.nlm.nih.gov/refseq/); UCSC Known Genes and Gencode (genome.ucsc.edu/index.html); and Gencode (www. gencodegenes.org/); as well as landmark publications (18-20). Each transcript was accurately identified by a specific exon or splice junction probe, and subsequently the hybridized arrays were washed, fixed and scanned using the Agilent DNA Microarray Scanner (model no., G2505C) and Feature Extraction software version 11.0.1.1 (Agilent Technologies, Inc.), which was used to analyze acquired array images. Quantile normalization and subsequent data processing were performed using the GeneSpring GX version 12.1 software (Agilent Technologies, Inc.). The microarray analysis was performed by KangChen Bio-tech, Inc. (Shanghai, China).

Gene ontology (GO) and pathway analysis. GO and pathway analysis were performed to identify the function and associated pathways of differentially expressed mRNAs. GO analysis was
Table I. Clinicopathological characteristics of patients with papillary thyroid carcinoma.

\begin{tabular}{lc} 
Characteristic & $\mathrm{n}$ \\
\hline Total & 36 \\
Age, years & \\
$<45$ & 15 \\
$\geq 45$ & 21 \\
Gender & \\
Male & 14 \\
Female & 22 \\
Tumor stage & \\
I + II & 24 \\
III + IV & 12 \\
Tumor size, cm & \\
$<1$ & 28 \\
$\geq 1$ & 8 \\
Lymph node involvement & \\
Yes & 15 \\
No & 21 \\
Multifocal & \\
Yes & 16 \\
No & 20 \\
\hline
\end{tabular}

derived from Gene Ontology (www.geneontology.org), which covers the following three domains: Biological process, cellular component and molecular function. The P-value denotes the significance of the GO term enrichment in the differentially expressed mRNA list (cut-off, $\mathrm{P}<0.05$ ). Pathway analysis for the differentially expressed mRNAs was based on the latest Kyoto Encyclopedia of Genes and Genomes (KEGG) pathways (www.genome.jp/kegg/). This analysis was performed in order to locate the significant enrichment pathways. The P-value denotes the significance of the pathway associated with the latest data from the KEGG database (cut-off, $\mathrm{P}<0.05$ ).

Classification and subgroup analysis. LncRNAs may be classified into different subgroups, including lncRNAs with enhancer-like function, antisense lncRNAs and large intergenic noncoding RNAs (lincRNAs).

To investigate the association between lncRNAs and their coding genes, IncRNAs with enhancer-like function were identified using the Gencode annotation of human genes (18). The selection of lncRNAs with enhancer-like function involved the exclusion of transcripts mapped to the exons and introns of annotated protein coding genes. These enhancer lncRNAs and the corresponding mRNAs $(<300 \mathrm{~kb})$ were integrated in order to study the potential function of the lncRNAs.

The function of antisense lncRNAs were identified based on the study by Faghihi and Wahlestedt (19). The present study integrated the differentially expressed antisense lncRNAs and corresponding sense mRNAs in order to deduce the function of the IncRNAs.

LincRNAs is a current hotspot of research. The function of lincRNAs were identified by the present study based on the 
Table II. Long noncoding RNA gene primers used for quantitative polymerase chain reaction.

\begin{tabular}{|c|c|c|c|}
\hline Primer name & Source & Primer sequence, $5^{\prime}-3^{\prime}$ & Product size, bp \\
\hline ENST00000503723 & Gencode & $\begin{array}{l}\text { F, TTTGAGTGATGACAGTTATATG } \\
\text { R, AACTTCTTAACCTACACCTTCT }\end{array}$ & 141 \\
\hline ENST00000423539 & Gencode & $\begin{array}{l}\text { F, CAAAGGTAATACAACTGTGAAG } \\
\text { R, TCTCTGCAAAAGGTTACATC }\end{array}$ & 87 \\
\hline uc003tab.3 & UCSC_knowngene & $\begin{array}{l}\text { F, TGCTTCTTCCATCCCTGCTTCC } \\
\text { R, TGGGAAGTTTGCGGCTCTGAA }\end{array}$ & 156 \\
\hline NR_073085 & RefSeq & $\begin{array}{l}\text { F, TTCTGGGGCTGCACCTTTGACT } \\
\text { R, TCACCTTCTCGGCTGCCTCTG }\end{array}$ & 155 \\
\hline ENST00000515275 & Gencode & $\begin{array}{l}\text { F, GGCAGTGTTCCAAATTAGATAT } \\
\text { R, AAGCAATTCTCAAAAGTCATG }\end{array}$ & 146 \\
\hline ENST00000570022 & Gencode & $\begin{array}{l}\text { F, CCGAGTGGAAGATGTCAGAGGAAACC } \\
\text { R, AACCGCTCGCACCTCAGGACTG }\end{array}$ & 177 \\
\hline uc003qef.1 & UCSC_knowngene & $\begin{array}{l}\text { F, CCCTCCATCTTGCCAAACTGCTGT } \\
\text { R, GGGTGGTGGTGAAAGGCAAAAGC }\end{array}$ & 180 \\
\hline ENST00000427243 & Gencode & $\begin{array}{l}\text { F, GCCAGTGTCTTCCAATGAGCTC } \\
\text { R, GCAGGTCTCAGGTCATCACAGG }\end{array}$ & 151 \\
\hline
\end{tabular}

Gencode, www.gencodegenes.org/; UCSC Gemome Bioinformatics, genome.ucsc.edu/index.html; RefSeq, www.ncbi.nlm.nih.gov/refseq/. F, forward; R, reverse.

study by Cabili et al (20). In order to deduce the function of these differentially expressed lincRNAs, the lincRNAs and corresponding nearby mRNAs underwent conjoint analysis.

Reverse transcription-quantitative polymerase chain reaction (RT-qPCR). RT-qPCR was performed to confirm the expression of lncRNAs identified by microarray analysis. In total, 8 lncRNAs (upregulated: ENST00000503723, ENST00000423539, uc003tab.3, NR_073085; downregulated: ENST00000515275, ENST00000570022, uc003qef.1, ENST00000427243) were selected to undergo qPCR analysis in 33 paired PTC and adjacent noncancerous samples. Total RNA was extracted from 33 frozen tumor specimens using TRIzol Reagent and subsequently reverse transcribed into cDNA using PrimeScript RT reagent kit (Invitrogen ${ }^{\mathrm{TM}}$ ), according to the manufacturer's protocol. DNase $(1 \mu \mathrm{l} ; 1 \mathrm{U} / \mu \mathrm{l})$ was purchased from Invitrogen ${ }^{\text {TM }}$. qPCR was performed using SYBR Green Real-Time PCR Master Mix (Invitrogen ${ }^{\mathrm{TM}}$ ), according to the manufacturer's protocol, on a MasterCycler RealPlex (Eppendorf, Hamburg, Germany). The following cycling conditions were used: $95^{\circ} \mathrm{C}$ for $2 \mathrm{~min}$; and $95^{\circ} \mathrm{C}$ for $15 \mathrm{sec}, 57^{\circ} \mathrm{C}$ for $15 \mathrm{sec}, 72^{\circ} \mathrm{C}$ for $1 \mathrm{~min}$ for a total 40 cycles. The primers used for the 8 lncRNAs are listed in Table II and were synthesized by Invitrogen ${ }^{\mathrm{TM}}$. Glyceraldehyde 3-phosphate dehydrogenase was used as a reference gene with the following primers: Forward, 5'-GAAAGCCTGCCGGTGACTAA-3' and reverse, 5'-AGGAAAAGCATCACCCGGAG-3'. For quantitative results, the expression of each IncRNA was represented as a fold change (FC) using $2^{-\Delta \Delta C q}$ (21). The experiment was repeated three times.

Statistical analysis. Statistical analysis was performed using Student's t-test for the comparison of two groups in the microarray. FC and the t-test were used to analyze the statistical significance of the microarray results. The false discovery rate was calculated to correct the P-value. The threshold value used to designate differentially expressed lncRNAs and mRNAs was a fold change of $\geq 2.0$ or $\leq 0.5, \mathrm{P}<0.05$. $\mathrm{P}<0.05$ was considered to indicate a statistically significant difference. Statistical analyses were performed with SPSS version 13 software (SPSS, Inc., Chicago, IL, USA).

\section{Results}

Expression profile of lncRNAs and mRNAs in PTC. Using microarray analysis, the present study examined the lncRNA and mRNA expression profiles in human paired PTC and adjacent noncancerous tissue samples (Exp vs. Ctrl). FC $(\mathrm{T} / \mathrm{N})$ and $\mathrm{P}$-value were calculated from the normalized expression. Microarray analysis revealed that 675 lncRNAs and 751 mRNAs were significantly differentially expressed in PTC samples compared with their adjacent noncancerous tissue samples $(\mathrm{FC} \geq 2.0 ; \mathrm{P}<0.05)$. Among the lncRNAs, 312 were upregulated and 363 were downregulated. Among the mRNAs, 499 were upregulated and 252 were downregulated. Hierarchical clustering analysis was used to arrange the differentially expressed lncRNAs into groups based on their expression levels. A dendrogram revealed the lncRNA expression patterns among samples ( $\mathrm{FC} \geq 4.0$; $\mathrm{P}<0.01$; Fig. 1 ). ENST00000503723 (FC=24.501809) was the most upregulated lncRNA, and ENST00000515275 ( FC=14.349726) was the most downregulated lncRNA.

Gene ontology analysis. Corresponding to the downregulated mRNAs, the total number of differentially expressed genes consisted of 159 genes involved in biological processes, 187 genes involved in cellular components and 170 genes involved in molecular functions. The highest enriched GOs 
A
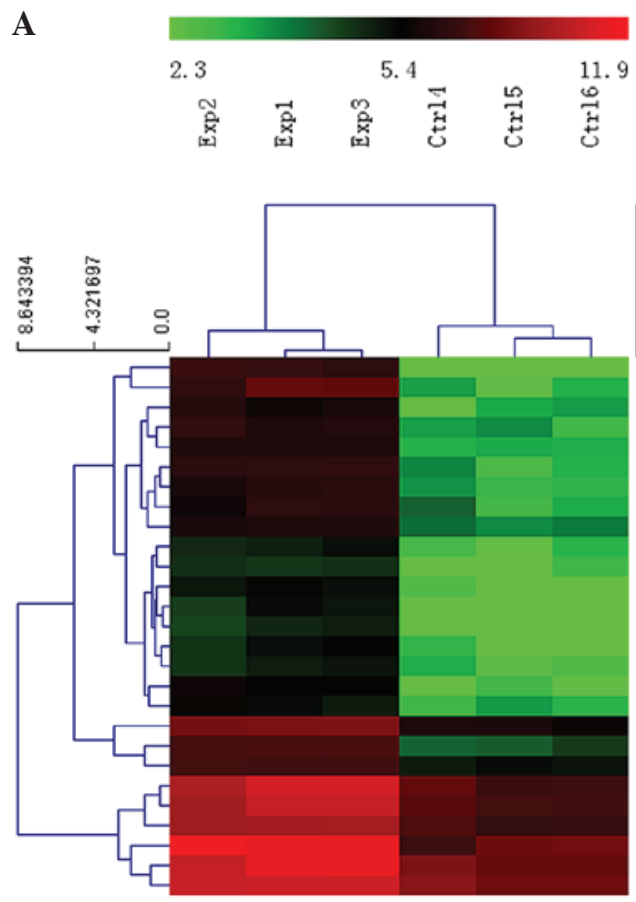

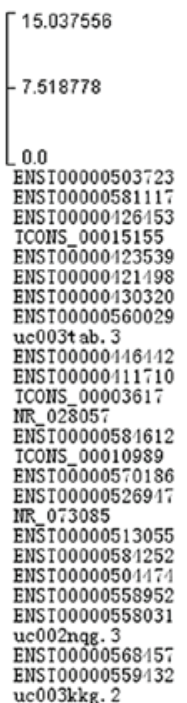

B
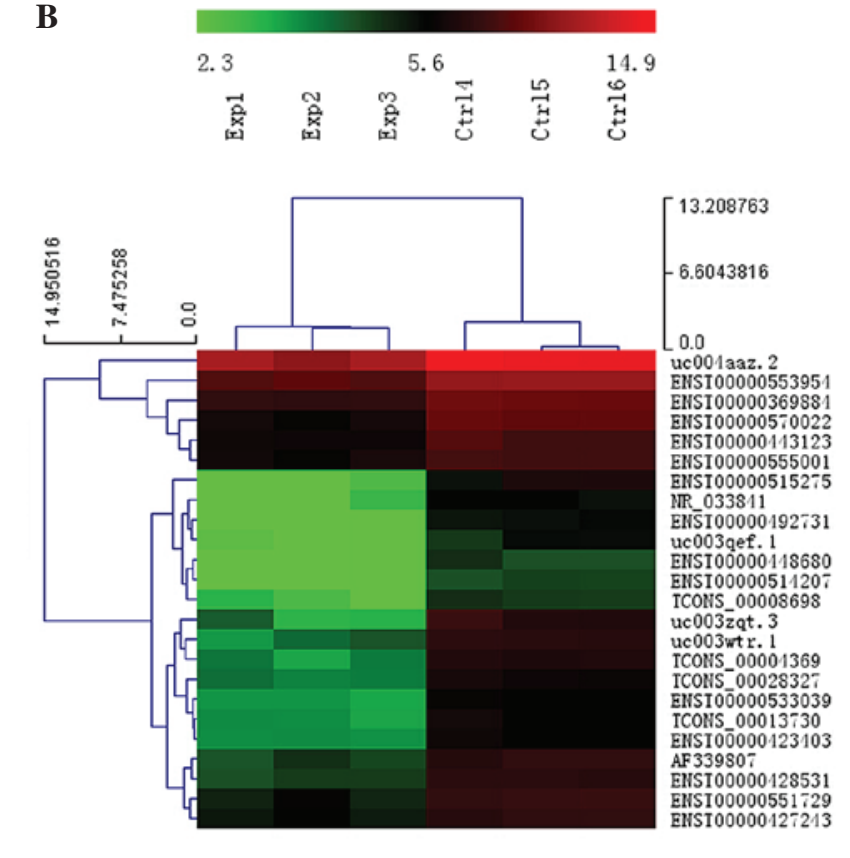

Figure 1. Hierarchical clustering dendrogram revealed a distinguishable lncRNA expression profile among samples (fold change $\geq 4.0$; $\mathrm{P}<0.01$ ). The heat map depicts (A) upregulated and (B) downregulated lncRNAs in PTC. Red indicates a high relative expression; green indicates a low relative expression. lncRNA, long noncoding RNAs; Exp, PTC samples; Ctrl, noncancerous adjacent samples; PTC, papillary thyroid carcinoma.

targeted by downregulated transcripts were negative regulation of transporter activity (biological process), myofibril (cellular component) and oxygen transporter activity (molecular function).

Corresponding to the upregulated mRNAs, the total number of differentially expressed genes consisted of 362 genes involved in biological processes, 376 genes involved in cellular components and 359 genes involved in molecular functions. The highest enriched GOs targeted by the upregulated transcripts were response to stimulus (biological process), plasma membrane (cellular component) and protein binding (molecular function).

Pathway analysis. Pathway analysis indicated that there were 7 downregulated pathways (Fig. 2A), and the most enriched network was 'Fc epsilon RI signaling pathway-Homo sapiens (human)', which was composed of 4 targeted genes. Furthermore, the analysis revealed that of the 29 upregulated pathways (Fig. 2B) identified, the most enriched network was 'p53 signaling pathway-Homo sapiens (human)', which was composed of 13 targeted genes.

Classification and subgroup analysis. LncRNA classification and subgroup analysis indicated that 7 pairs of enhancer-like lncRNA-mRNA (Table III), 9 pairs of antisense lncRNA-mRNA (Table IV) and 45 pairs of lincRNA-mRNA (Table V) were differentially expressed between PTC and their paired adjacent noncancerous samples.

$R T$-qPCR results. To verify the microarray data, 8 differentially expressed lncRNAs, including 4 upregulated (ENST00000503723, ENST00000423539, uc003tab.3 and NR_073085) and 4 downregulated (ENST00000515275,
ENST00000570022, uc003qef.1 and ENST00000427243) lncRNAs, were randomly selected for RT-qPCR in 33 pairs of PTC and adjacent noncancerous tissues. The results were consistent with the microarray data (Fig. 3).

\section{Discussion}

PTC is a common malignant tumor of the endocrine system that occurs more often in women than men (22). The incidence of PTC has increased globally for several decades. The tumorigenesis of PTC is a complex biological process characterized by various molecular abnormalities. Over the past few decades, the molecular mechanism of PTC has been extensively investigated; however, the exact pathogenesis of this disease remains unclear. Increasing evidence suggests that lncRNAs may be significant in regulating gene expression. LncRNA have gained considerable attention and have become the frontiers of ncRNA research. Although lncRNAs were initially indicated to be transcriptional noise, studies suggest that they may be key in cellular development and human diseases $(23,24)$, including lnc-IL7R in the inflammatory response (25), and HOTAIR and Gas5 in breast tumors and metastases $(16,26,27)$. Aberrant expression of lncRNAs were also identified in the present study in PTC using microarray data, including HOTAIR, which was downregulated; however, this was not statistical significant. Of all the functions of lncRNAs, the most important is their involvement in tumorigenesis. However, the role of lncRNAs in tumor pathogenesis and development is less well characterized.

The present study investigated the lncRNA expression profiles of PTC using microarray analysis and revealed that lncRNA expression levels were altered compared to adjacent noncancerous tissues. From the microarray expression 
A

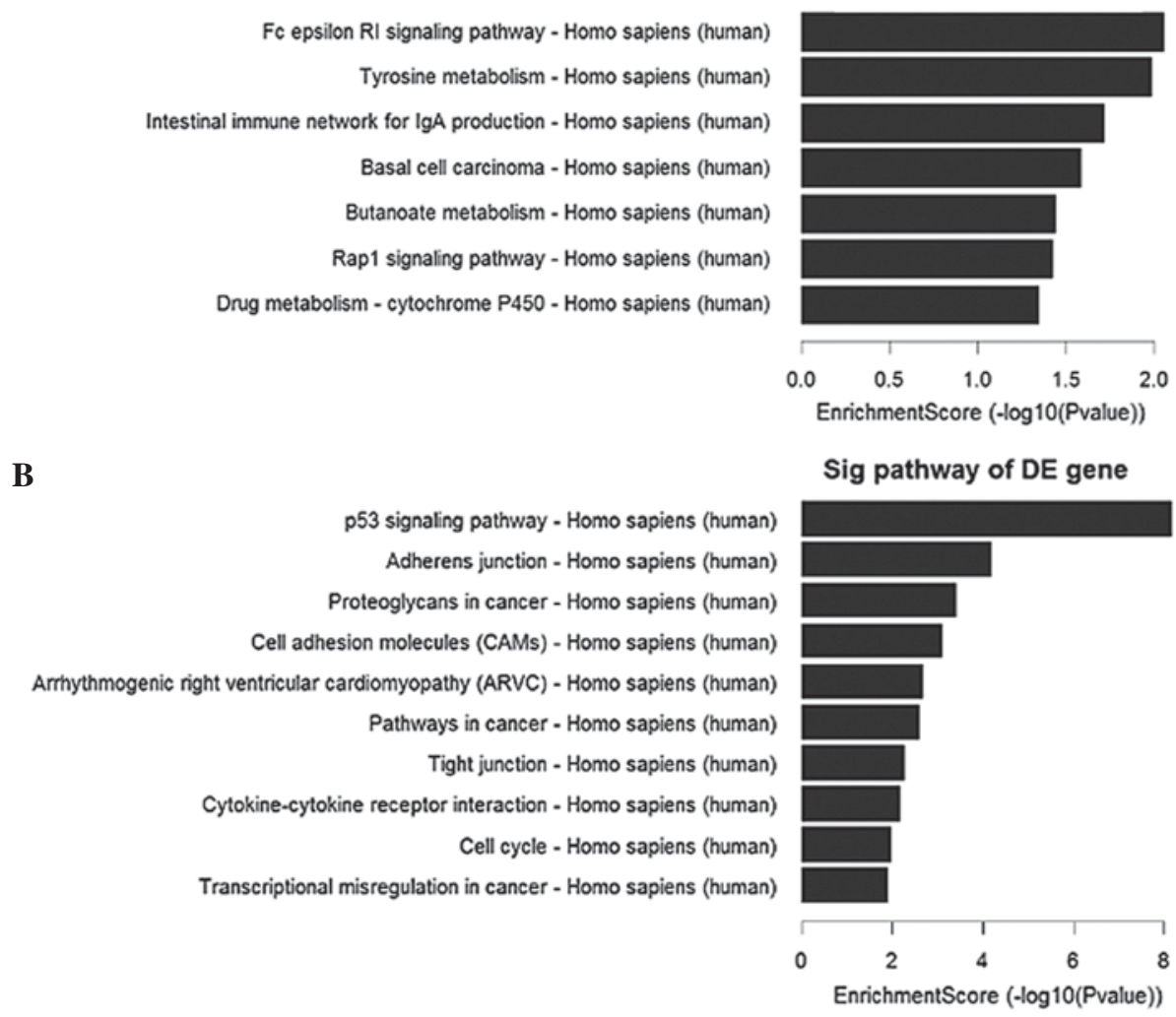

Figure 2. Pathway analysis of mRNAs between the papillary thyroid carcinoma and noncancerous adjacent samples. (A) The 7 downregulated pathways identified. (B) The top 10 out of 29 upregulated pathways identified. DE, differentially expressed; sig, signaling.

A

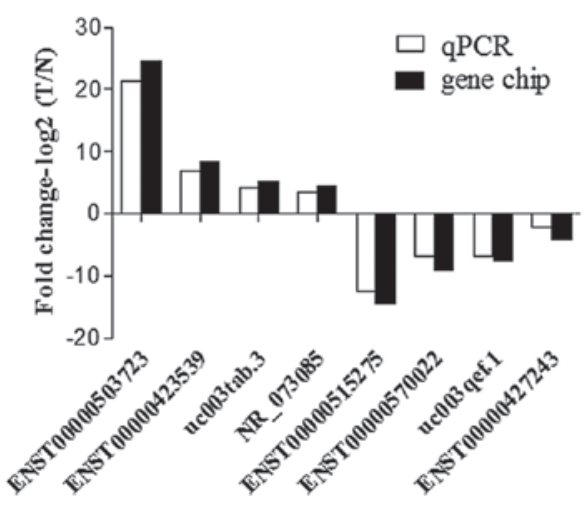

B

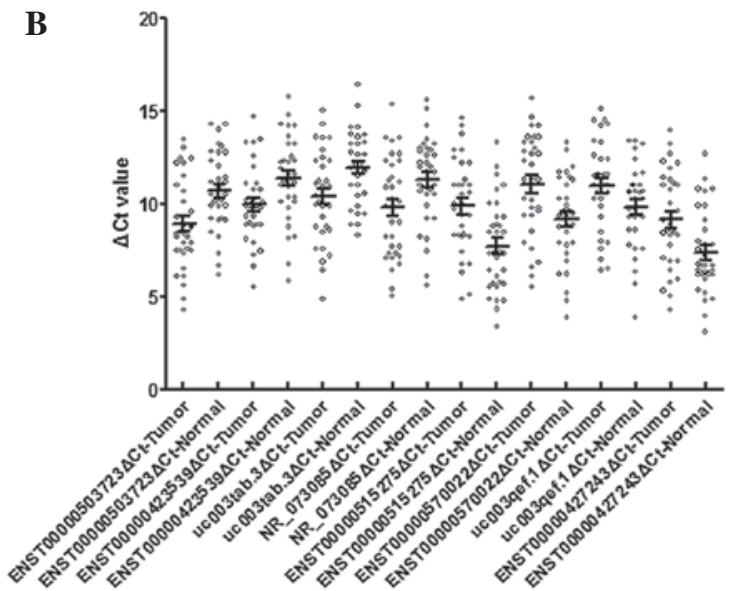

Figure 3. Differential expression of lncRNAs identified by the microarray analysis was validated by RT-qPCR. (A) Comparison between microarray and RT-qPCR results. In total, 8 differentially expressed lncRNAs identified by the microarray were validated by RT-qPCR. The heights of the columns represent the log-transformed median fold changes $(\mathrm{T} / \mathrm{N})$ in the expression of 33 paired PTC and adjacent noncancerous samples $(\mathrm{P}<0.01)$. The RT-qPCR results and microarray data were consistent. (B) Distribution of 8 lncRNAs in 33 paired PTC and adjacent noncancerous samples (P<0.01). PTC, papillary thyroid carcinoma.

profiles, 312 upregulated lncRNAs and 363 downregulated lncRNAs were significantly differentially expressed in all PTC samples. The most upregulated lncRNA was ENST00000503723, and the most downregulated lncRNA was ENST00000515275. This result suggests that ENST00000503723 and ENST00000515275 may contribute to the development of PTC; however, additional study concerning the biological function of these two lncRNAs requires confirmation. To validate the microarray profiling expression data, the expression of 8 randomly selected lncRNAs were examined in PTC and adjacent noncancerous samples by RT-qPCR. The RT-qPCR results confirmed the data from the microarray.

The present results demonstrated an altered expression profile of lncRNAs in PTC. Additional analysis by the present study highlighted that numerous genes associated with these lncRNAs were associated with various types of cancer, including ENST00000427243, uc003qef.1 and NR_073085. 
Table III. Enhancer-like lncRNA-mRNA and nearby genes.

\begin{tabular}{|c|c|c|c|c|c|}
\hline Seqname & $\begin{array}{c}\text { Regulation, } \\
\text { lncRNAs }\end{array}$ & Genome assocation & Nearby gene seq. & Nearby gene & $\begin{array}{c}\text { Regulation, } \\
\text { mRNAs }\end{array}$ \\
\hline ENST00000418242 & Up & Downstream & NM_001122674 & $\mathrm{ABCD} 3$ & Up \\
\hline ENST00000423539 & Up & Upstream & NM_020651 & PELI1 & Up \\
\hline ENST00000429328 & Up & Upstream & NM_178134 & CYP4Z1 & Down \\
\hline ENST00000430945 & Up & Downstream & NM_014057 & OGN & Down \\
\hline ENST00000430945 & Up & Upstream & NM_004560 & ROR2 & Down \\
\hline ENST00000442526 & Down & Upstream & NM_018590 & CSGALNACT2 & Up \\
\hline NR_024160 & Down & Upstream & NM_001937 & DPT & Down \\
\hline
\end{tabular}

ABCD3, ATP binding cassette subfamily D member 3; PELI1, pellino E3 ubiquitin protein ligase 1; CYP4Z1, cytochrome P450 family 4 subfamily Z member 1; OGN, osteoglycin; ROR2, receptor tyrosine kinase like orphan receptor 2; CSGALNAXT2, chondroitin sulfate $\mathrm{N}$-acetylgalactosaminyltransferase 2; DPT, dermatopontin; Seq., sequence; lncRNA, long non-coding RNA.

Table IV. Antisense lncRNA-mRNA and nearby genes.

\begin{tabular}{|c|c|c|c|c|c|}
\hline Seqname & $\begin{array}{c}\text { Regulation, } \\
\text { lncRNAs }\end{array}$ & Genome assocaition & Nearby gene seq. & Nearby gene & $\begin{array}{c}\text { Regulation, } \\
\text { mRNAs }\end{array}$ \\
\hline ENST00000506160 & Down & Intronic antisense & NM_000667 & ADH1A & Down \\
\hline ENST00000541892 & $\mathrm{Up}$ & Intronic antisense & NM_001001395 & LMO3 & Up \\
\hline ENST00000550214 & Up & Intronic antisense & NM_001651 & AQP5 & $\mathrm{Up}$ \\
\hline NR_036580 & Up & Intronic antisense & NM_001178036 & DPP10 & Up \\
\hline ENST00000418335 & Up & Natural antisense & NM_001935 & DPP4 & Up \\
\hline ENST00000419425 & Up & Natural antisense & NM_006449 & CDC42EP3 & $\mathrm{Up}$ \\
\hline ENST00000512486 & Up & Natural antisense & NM_014899 & RHOBTB3 & $\mathrm{Up}$ \\
\hline NR_033252 & Down & Natural antisense & NM_001482 & GATM & Down \\
\hline uc001pnn.3 & Down & Natural antisense & NM_181351 & NCAM1 & Down \\
\hline
\end{tabular}

ADH1A, alcohol dehydrogenase 1A (class I), alpha polypeptid; LMO3, LIM domain only 3; AQP5, aquaporin 5; DPP, dipeptidyl peptidase; CDC42EP3, cell division cycle 42 effector protein 3; RHOBTB3, Rho related BTB domain containing 3; GATM, glycine amidinotransferase; NCAM1, neural cell adhesion molecule 1; Seq., sequence; lncRNA, long non-coding RNA.

ENST00000427243 was significantly downregulated in PTC and was identified to be associated with breast cancer anti-estrogen resistance protein 3 (BCAR3) gene. BCAR3 overexpression results in enhanced cancer cell migration and colocalization (28). By contrast, BCAR3 depletion inhibits cancer cell migration and invasion (29). In addition, BCAR3 functions as a positive regulator in invasive breast cancer cells (30) and acts as a putative suppressor of breast cancer progression (31). Therefore, there may be an association between ENST00000427243 lncRNA and BCAR3 in the development of PTC, and BCAR3 may aid in the understanding of PTC and lncRNAs at the transcriptional level.

uc003qef.1 was also identified to be significantly downregulated in the present results. Its associated gene was eyes absent homolog 4 (EYA4). Decreased EYA4 expression is not only associated with poor survival in sporadic lung cancer, but also EYA4 single-nucleotide polymorphisms have been associated with an increased familial cancer risk (32). The present study concludes that uc003qef.1 may play an important role with EYA4 in the familial cancer risk of PTC.
NR_073085 was identified to be significantly upregulated in PTC samples by the present study. NR_073085 was associated with cysteine-rich protein 2 (CRIP2), which was identified as an important candidate gene capable of functionally suppressing tumor formation (33). CRIP2 is significantly downregulated in tumors, and an overexpression of CRIP2 may induce cancer cell apoptosis (33). Furthermore, CRIP2 acts as a transcriptional repressor of the nuclear factor- $\kappa \mathrm{B}$-mediated proangiogenic cytokine expression, and thereby functionally inhibits tumor formation and angiogenesis (34). NR_073085 may be significant in the regulation of CRIP2 during tumor progression. The association between NR_073085 and PTC requires additional experimental verification.

The microarray-based profiling performed by the present study identified $751 \mathrm{mRNAs}$ with a differential expression in PTC. GO analysis and pathway analysis indicated that there were 7 downregulated and 29 upregulated pathways in PTC compared with adjacent non-tumorous tissues. Among the upregulated pathways, the p53 signaling pathway was the most enriched network. Lan et al (35) also demonstrated that the 
Table V. Large intergenic noncoding RNA-mRNA and nearby genes.

\begin{tabular}{|c|c|c|c|c|c|}
\hline Seq. name & $\begin{array}{c}\text { Regulation, } \\
\text { IncRNAs }\end{array}$ & Genome association & Nearby gene seq. & Nearby gene & $\begin{array}{c}\text { Regulation, } \\
\text { mRNAs }\end{array}$ \\
\hline TCONS_00001038 & Down & Upstream & NM_001122674 & ABCD3 & Up \\
\hline TCONS_00003617 & Up & Upstream & NM_022823 & FNDC4 & Up \\
\hline TCONS_00003757 & Up & Upstream & NM_001042437 & ST3GAL5 & Up \\
\hline TCONS_00003757 & Up & Upstream & NM_198843 & SFTPB & Up \\
\hline TCONS_00004186 & Up & Downstream & NM_022823 & FNDC4 & Up \\
\hline TCONS_00004538 & Up & Downstream & NM_012223 & MYO1B & Up \\
\hline TCONS_00004570 & Up & Downstream & NM_001079526 & IKZF2 & Up \\
\hline TCONS_00005191 & Down & Downstream & NM_001042437 & ST3GAL5 & Up \\
\hline TCONS_00005191 & Down & Downstream & NM_198843 & SFTPB & Up \\
\hline TCONS_00005192 & Down & Downstream & NM_001042437 & ST3GAL5 & Up \\
\hline TCONS_00005192 & Down & Downstream & NM_198843 & SFTPB & Up \\
\hline TCONS_00005258 & Down & Upstream & NM_000888 & ITGB6 & Up \\
\hline TCONS_00005258 & Down & Upstream & NM_001007267 & PLA2R1 & Down \\
\hline TCONS_00005258 & Down & Upstream & NM_007366 & PLA2R1 & Down \\
\hline TCONS_00005259 & Down & Upstream & NM_000888 & ITGB6 & Up \\
\hline TCONS_00005259 & Down & Upstream & NM_001007267 & PLA2R1 & Down \\
\hline TCONS_00005259 & Down & Upstream & NM_007366 & PLA2R1 & Down \\
\hline TCONS_00006023 & Up & Upstream & NM_004635 & MAPKAPK3 & Up \\
\hline TCONS_00006284 & Down & Upstream & NM_014996 & PLCH1 & Down \\
\hline TCONS_00006733 & Down & Downstream & ENST00000072516 & IL1RAP & Up \\
\hline TCONS_00006733 & Down & Downstream & NM_134470 & IL1RAP & Up \\
\hline TCONS_00008649 & Down & Downstream & ENST00000265000 & GALNT7 & Up \\
\hline TCONS_00008698 & Down & Downstream & ENST00000448662 & SORBS2 & Down \\
\hline TCONS_00008698 & Down & Downstream & NM_001145670 & SORBS2 & Down \\
\hline TCONS_00008698 & Down & Downstream & NM_003603 & SORBS2 & Down \\
\hline TCONS_00008738 & Down & Downstream & NM_006527 & SLBP & Up \\
\hline TCONS_00008739 & Down & Downstream & NM_006527 & SLBP & Up \\
\hline TCONS_00010526 & Up & Upstream & NM_001112724 & STK32A & Up \\
\hline TCONS_00010526 & Up & Upstream & NM_145001 & STK32A & Up \\
\hline TCONS_00010794 & Up & Upstream & NM_004887 & CXCL14 & Up \\
\hline TCONS_00012533 & Up & Upstream & NM_001122769 & LCA5 & Up \\
\hline TCONS_00012535 & Up & Upstream & NM_001122769 & LCA5 & Up \\
\hline TCONS_00014681 & Up & Downstream & NM_020130 & C8orf4 & Up \\
\hline TCONS_00016073 & Down & Upstream & NM_014057 & OGN & Down \\
\hline TCONS_00016117 & Up & Upstream & NM_021224 & ZNF462 & Up \\
\hline TCONS_00016354 & Up & Upstream & NM_000700 & ANXA1 & $\mathrm{Up}$ \\
\hline TCONS_00018333 & Up & Upstream & NM_004419 & DUSP5 & Up \\
\hline TCONS_00018333 & Up & Upstream & NM_005962 & MXI1 & Down \\
\hline TCONS_00019600 & Down & Upstream & NM_001584 & MPPED2 & Down \\
\hline TCONS_00019602 & Down & Downstream & NM_001584 & MPPED2 & Down \\
\hline TCONS_00020396 & Down & Downstream & NM_181847 & AMIGO2 & Up \\
\hline TCONS_00024026 & Down & Downstream & NM_024708 & ASB7 & Up \\
\hline TCONS_00027184 & Down & Upstream & NM_031209 & QTRT1 & Down \\
\hline TCONS_00027673 & Down & Upstream & NM_031209 & QTRT1 & Down \\
\hline TCONS_00029753 & Up & Upstream & NM_001193414 & TUBA8 & Down \\
\hline
\end{tabular}

Seq., sequence; lncRNA, long non-coding RNA.

p53 signaling pathway was differentially expressed in PTC in a mRNA expression profile study. It is known that p53 plays an important tumor suppressive role during thyroid carcinoma progression (36). Signaling pathways that use lncRNAs to regulate the growth and metastasis of tumors require additional study to be identified.

Furthermore, IncRNA classification and subgroup analysis performed by the present study indicated 61 pairs 
of IncRNA-mRNA were differentially expressed in PTC. Certain nearby proteins of these lncRNAs were associated with inflammation. It is commonly accepted that cancer is associated with inflammation, and an intense immune infiltrate is often observed with PTC, which may be critical in the regulation of carcinogenesis and carcinoma progression (37). The present study identified ENST00000423539 as a significantly upregulated enhancer lncRNA in PTC, and the nearby protein was E3 ubiquitin-protein ligase pellino homolog 1 (Peli1). Peli1 regulates Toll-like receptor pathway signaling by promoting the degradation of tumor necrosis factor receptor-associated factor 3 , which is a potent inhibitor of mitogen-activated protein kinase activation and gene induction (38). In addition, Peli1 is a target of microRNA-21 (39), which is significantly downregulated in PTC tissues (40). ENST00000423539 may exert its functions through interactions with coding transcripts in PTC. The inflammatory pathways of cancer-associated inflammation is critical for the identification of novel target molecules that may lead to improved diagnosis and treatment.

In conclusion, the present study demonstrated the existence of a significantly altered lncRNA and mRNA expression profile in PTC. Dysregulation of these lncRNAs may be important in the development, invasion and metastasis of tumors. Additional study is required to determine whether these alterations are indirectly due to genotype, familial cancer risk, inflammatory states or a combination of these cancer risk factors. Further molecular mechanism studies may aid in the elucidation of the exact roles of these lncRNAs in PTC.

\section{Acknowledgements}

The authors would like to thank all the donors that donated to the Microarray Service at KangChen Bio-tech, Inc.

\section{References}

1. La Vecchia C, Malvezzi M, Bosetti C, Garavello W, Bertuccio P, Levi F and Negri E: Thyroid cancer mortality and incidence: A global overview. Int J Cancer 136: 2187-2195, 2015.

2. Siegel R, Ma J, Zou Z and Jemal A: Cancer statistics, 2014. CA Cancer J Clin 64: 9-29, 2014

3. Yang L, Zheng R, Wang N, Zhang S and Chen W: Analysis of incidence and mortality of thyroid cancer in China, 2010 Zhonghua Yu Fang Yi Xue Za Zhi 48: 663-668, 2014 (In Chinese).

4. Pelizzo MR, Merante Boschin I, Toniato A, Pagetta C, Casal Ide E, Mian C and Rubello D: Diagnosis, treatment, prognostic factors and long-term outcome in papillary thyroid carcinoma. Minerva Endocrinol 33: 359-379, 2008.

5. Richards ML: Familial syndromes associated with thyroid cancer in the era of personalized medicine. Thyroid 20: 707-713, 2010.

6. Galleani J, Miranda C, Pierotti MA and Greco A: H2AX phosphorylation and kinetics of radiation-induced DNA double strand break repair in human primary thyrocytes. Thyroid 19: 257-264, 2009.

7. Leux C, Truong T, Petit C, Baron-Dubourdieu D and Guénel P: Family history of malignant and benign thyroid diseases and risk of thyroid cancer: A population-based case-control study in New Caledonia. Cancer Causes Control 23: 745-755, 2012.

8. Santos JC, Bastos AU, Cerutti JM and Ribeiro ML: Correlation of MLH1 and MGMT expression and promoter methylation with genomic instability in patients with thyroid carcinoma. BMC Cancer 13: 79, 2013

9. Neta G, Brenner AV, Sturgis EM, Pfeiffer RM, Hutchinson AA, Aschebrook-Kilfoy B, Yeager M, Xu L, Wheeler W, Abend $\mathrm{M}$, et al: Common genetic variants related to genomic integrity and risk of papillary thyroid cancer.Carcinogenesis 32: 1231-1237, 2011
10. Taft RJ, Pang KC, Mercer TR, Dinger M and Mattick JS: Noncoding RNAs: Regulators of disease. J Pathol 220: 126-139, 2010.

11. Djebali S, Davis CA, Merkel A, Dobin A, Lassmann T, Mortazavi A, Tanzer A, Lagarde J, Lin W, Schlesinger F, et al: Landscape of transcription in human cells. Nature 489: 101-108, 2012.

12. Jendrzejewski J, He H, Radomska HS, Li W, Tomsic J, Liyanarachchi S, Davuluri RV, Nagy R and de la Chapelle A: The polymorphism rs 944289 predisposes to papillary thyroid carcinoma through a large intergenic noncoding RNA gene of tumor suppressor type. Proc Natl Acad Sci USA 109: 8646-8651, 2012.

13. Wang Y, Guo Q, Zhao Y, Chen J, Wang S, Hu J and Sun Y: BRAF-activated long non-coding RNA contributes to cell proliferation and activates autophagy in papillary thyroid carcinoma. Oncol Lett 8: 1947-1952, 2014.

14. Dinger ME, Pang KC, Mercer TR, Crowe ML, Grimmond SM and Mattick JS: NRED: A database of long noncoding RNA expression. Nucleic Acids Res 37 (Database Issue): D122-D126, 2009.

15. Ponting CP, Oliver PL and Reik W: Evolution and functions of long noncoding RNAs. Cell 136: 629-641, 2009.

16. Gupta RA, Shah N, Wang KC, Kim J, Horlings HM, Wong DJ, Tsai MC, Hung T, Argani P, Rinn JL, et al: Long non-coding RNA HOTAIR reprograms chromatin state to promote cancer metastasis. Nature 464: 1071-1076, 2010.

17. Zhu M, Chen Q, Liu X, Sun Q, Zhao X, Deng R, Wang Y, Huang J, Xu M, Yan J and Yu J: lncRNA H19/miR-675 axis represses prostate cancer metastasis by targeting TGFBI. FEBS J 281: 3766-3775, 2014.

18. Ørom UA, Derrien T, Beringer M, Gumireddy K, Gardini A, Bussotti G, Lai F, Zytnicki M, Notredame C, Huang Q, et al: Long noncoding RNAs with enhancer-like function in human cells. Cell 143: 46-58, 2010.

19. Faghihi MA and Wahlestedt C: Regulatory roles of natural antisense transcripts. Nat Rev Mol Cell Biol 10: 637-643, 2009.

20. Cabili MN, Trapnell C, Goff L, Koziol M, Tazon-Vega B, Regev A and Rinn JL: Integrative annotation of human large intergenic noncoding RNAs reveals global properties and specific subclasses. Genes Dev 25: 1915-1927, 2011.

21. Xu G, Chen J, Pan Q, Huang K, Pan J, Zhang W, Chen J, Yu F, Zhou $\mathrm{T}$ and Wang Y: Long noncoding RNA expression profiles of lung adenocarcinoma ascertained by microarray analysis. PLoS One 9: e104044, 2014.

22. Kweon SS, Shin MH, Chung IJ, Kim YJ and Choi JS:Thyroid cancer is the most common cancer in women, based on the data from population-based cancer registries, South Korea. Jpn J Clin Oncol 43: 1039-1046, 2013.

23. Wilusz JE, Sunwoo H and Spector DL: Long noncoding RNAs: Functional surprises from the RNA world. Genes Dev 23: 1494-1504, 2009.

24. Mercer TR, Dinger ME and Mattick JS: Long non-coding RNAs: Insights into functions. Nat Rev Genet 10: 155-159, 2009.

25. Cui H, Xie N, Tan Z, Banerjee S, Thannickal VJ, Abraham E and Liu G: The human long noncoding RNA lnc-IL7R regulates the inflammatory response. Eur J Immunol 44: 2085-2095, 2014.

26. Bhan A, Hussain I, Ansari KI, Bobzean SA, Perrotti LI and Mandal SS: Bisphenol-A and diethylstilbestrol exposure induces the expression of breast cancer associated long noncoding RNA HOTAIR in vitro and in vivo. J Steroid Biochem Mol Biol 141: 160-170, 2014.

27. Mourtada-Maarabouni M, Pickard MR, Hedge VL, Farzaneh F and Williams GT: GAS5, a non-protein-coding RNA, controls apoptosis and is downregulated in breast cancer. Oncogene 28: 195-208, 2009.

28. Wallez Y, Riedl SJ and Pasquale EB: Association of the breast cancer antiestrogen resistance protein 1 (BCAR1) and BCAR3 scaffolding proteins in cell signaling and antiestrogen resistance. J Biol Chem 289: 10431-10444, 2014.

29. Schrecengost RS, Riggins RB, Thomas KS, Guerrero MS and Bouton AH: Breast cancer antiestrogen resistance-3 expression regulates breast cancer cell migration through promotion of p130Cas membrane localization and membrane ruffling. Cancer Res 67: 6174-6182, 2007.

30. Wilson AL, Schrecengost RS, Guerrero MS, Thomas KS and Bouton AH: Breast cancer antiestrogen resistance 3 (BCAR3) promotes cell motility by regulating actin cytoskeletal and adhesion remodeling in invasive breast cancer cells. PLoS One 8: e65678, 2013. 
31. Guo J, Canaff L, Rajadurai C, Fils-Aimé N, Tian J, Dai M, Korah J, Villatoro M, Park M, Ali S and Lebrun JJ: Breast cancer anti-estrogen resistance-3 inhibits transforming growth factor- $\beta /$ Smad signaling and associates with favorable breast cancer disease outcomes. Breast Cancer Res 16: 476, 2014.

32. Wilson IM, Vucic EA, Enfield KS, Thu KL, Zhang YA, Chari R, Lockwood WW, Radulovich N, Starczynowski DT, Banáth JP, et al: EYA4 is inactivated biallelically at a high frequency in sporadic lung cancer and is associated with familial lung cancer risk. Oncogene 33: 4464-4473, 2014.

33. Lo PH, Ko JM, Yu ZY, Law S, Wang LD, Li JL, Srivastava G, Tsao SW, Stanbridge EJ and Lung ML: The LIM domain protein, CRIP2, promotes apoptosis in esophageal squamous cell carcinoma. Cancer Lett 316: 39-45, 2012.

34. Cheung AK, Ko JM, Lung HL, Chan KW, Stanbridge EJ, Zabarovsky E, Tokino T, Kashima L, Suzuki T, Kwong DL, et al: Cysteine-rich intestinal protein 2 (CRIP2) acts as a repressor of NF-kappaB-mediated proangiogenic cytokine transcription to suppress tumorigenesis and angiogenesis. Proc Natl Acad Sci USA 108: 8390-8395, 2011.

35. Lan X, Zhang H, Wang Z, Dong W, Sun W, Shao L, Zhang T and Zhang D: Genome-wide analysis of long noncoding RNA expression profile in papillary thyroid carcinoma. Gene 569 $109-117,2015$
36. McFadden DG, Vernon A, Santiago PM, Martinez-McFaline R, Bhutkar A, Crowley DM, McMahon M, Sadow PM and Jacks T: p53 constrains progression to anaplastic thyroid carcinoma in a Braf-mutant mouse model of papillary thyroid cancer. Proc Natl Acad Sci USA 111: E1600-E1609, 2014.

37. Liotti F, Visciano $C$ and Melillo RM: Inflammation in thyroid oncogenesis. Am J Cancer Res 2: 286-297, 2012.

38. Xiao Y, Jin J, Chang M, Chang JH, Hu H, Zhou X, Brittain GC, Stansberg C, Torkildsen $\varnothing$, Wang X, et al: Peli1 promotes microglia-mediated CNS inflammation by regulating Traf3 degradation. Nat Med 19: 595-602, 2013.

39. Marquez RT, Wendlandt E, Galle CS, Keck K, McCaffrey AP: MicroRNA-21 is upregulated during the proliferative phase of liver regeneration, targets Pellino-1, and inhibits NF-kappaB signaling. Am J Physiol Gastrointest Liver Physiol 298: G535-G541, 2010.

40. Huang Y, Liao D, Pan L, Ye R, Li X, Wang S, Ye C and Chen L: Expressions of miRNAs in papillary thyroid carcinoma and their associations with the BRAFV600E mutation. Eur J Endocrinol 168: 675-681, 2013. 